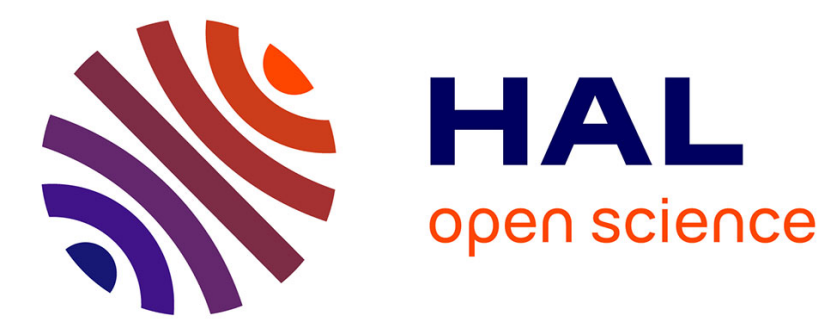

\title{
Note de fin de texte
}

Thierry Wendling

\section{To cite this version:}

Thierry Wendling. Note de fin de texte: La note de fin de texte et ses héros. Ethnologie française, 2021, Vol. 51 (1), pp.95-96. 10.3917/ethn.211.0095 . hal-03332485

\section{HAL Id: hal-03332485 \\ https://hal.science/hal-03332485}

Submitted on 2 Sep 2021

HAL is a multi-disciplinary open access archive for the deposit and dissemination of scientific research documents, whether they are published or not. The documents may come from teaching and research institutions in France or abroad, or from public or private research centers.
L'archive ouverte pluridisciplinaire HAL, est destinée au dépôt et à la diffusion de documents scientifiques de niveau recherche, publiés ou non, émanant des établissements d'enseignement et de recherche français ou étrangers, des laboratoires publics ou privés. 


\section{La note de fin de texte et ses héros}

Thierry Wendling

[version du 10.4.2020 déposée sur HAL en septembre 2021 en raison d'une exigence administrative ; l'article, paru dans Ethnologie française, LI, 2021, 1, p. 97-98, constitue la seule version intégrale et authentique]

Tout discours, et même toute particule de discours, s'inscrit dans une rhétorique. À cet égard, la note de bas de page ou de fin de texte signale en SHS le passage de l'essai à la thèse, du roman à l'affirmation scientifique. Remarquons tout de suite qu'il n'y a là aucune logique obligée. On pourrait imaginer que des romanciers mobilisent ce dispositif pour rendre compte de la multiplicité des voix, des regards, des interprétations et que tout imprimé qui se donne comme fiction apparaisse comme une mosaïque de briques textuelles encastrées, pouvant à l'occasion s'approcher, sous l'angle de la forme, du folio d'un Talmud, avec ses différents blocs, ou d'un manuel du jeu d'échecs déclinant les innombrables variantes d'une ouverture.

Depuis Fenimore Cooper (dont Le Dernier des Mohicans est enrichi de précisions historiques et culturelles), ou même d'autres avant lui, quelques rares romanciers ont usé du procédé mais ce deuxième niveau d'écriture semble, dans l'ensemble, réservé à des pas-tout-à-fait auteurs, à des auteurs d'un deuxième temps qui viennent commenter à leur manière une œuvre. C'est le traducteur qui, tout à son effort de rendre au plus près les idées, la sonorité, les images que véhicule le texte, bute contre un obstacle paradoxal $^{1}$. C'est le responsable d'une édition critique si heureux de pouvoir témoigner que, ce jour-là, Proust s'était couché fort tard. Et puis bien sûr, on l'a avec raison souligné, c'est l'historien, le sociologue, l'ethnologue qui fait science ${ }^{2}$.

\footnotetext{
${ }^{1}$ En français dans l'original (lui-même écrit dans une langue romane issue du latin vulgaire, mais enrichie d'éléments gaulois, germaniques, arabes, wolof, chinois, tupi...). (N.d.T.).

${ }^{2}$ Exceptionnelles sont les contributions d'Ethnologie française qui ne sont pas assorties de notes et d'une bibliographie, mais une réflexion d'ordre épistémologique ne semble affleurer que dans les comptes rendus d'ouvrages : on vante la précision, l'abondance des notes ; inversement, on critique l'absence de tout appareil critique ; et, à l'occasion, on regrette que soient « rejetées en note des observations pourtant très intéressantes ». J'ignore le nombre moyen de notes par article, ni le record absolu. Au vu d'un article paru en 1974, je conseillerai néanmoins aux lecteurs de se méfier d'une première note annonçant modestement « Ce n'est pas ici le lieu de citer toutes ces recherches ni d'en
} 
Mais dans la fascination que suscite idéalement tout récit, tout discours, la note de bas de page surgit comme une de ces croisées de chemin où le héros d'un conte doit prendre une décision. Le petit chiffre placé en exposant stoppe brutalement la lecture. Il enjoint de faire un choix. Jusqu'alors le lecteur se laissait porter par le texte, adaptant juste le rythme de son parcours à l'intérêt du paysage décrit, à la difficulté des cols à passer, s'autorisant parfois à dévaler quelques pentes herbeuses un peu ennuyeuses.

Or, dans la randonnée d'une lecture, l'appel de note peut être vécu comme une technique cognitive car il exerce l'activité critique. Il y a de l'impertinence dans la note. Certes, du point de vue de l'auteur, elle peut être considérée comme une digression ou un complément qui témoigne de sa volonté d'explicitation, de précision, de nuance. Mais pour celui ou celle qui progresse dans la forêt des mots, la note relève presque de l'impolitesse et c'est cette insolence, cette provocation (me liras-tu? semble-t-elle toujours demander), qui participe au développement d'un jugement personnel, aussi mineur soit-il. Il faut dire qu'elle surgit n'importe où, au débotté ; rien ne la laisse présager, ni annonce de plan scolaire, ni technique littéraire plus subtile. Elle déboule souvent comme une maroufle au beau milieu d'un propos. Il nous faut nous rappeler notre expérience commune de lecteurs, cette hésitation qui nous saisit alors : vais-je m'engager immédiatement dans cette voie de traverse, ou attendre la fin de la phrase, du paragraphe? Et d'abord, vais-je seulement accepter de me détourner pour explorer cette note ? Si je décide de la négliger, un remords me poursuit, comme le caillou dans la chaussure que le marcheur veut ignorer (n'ai-je pas raté un exemple, un argument, un repère bibliographique qui s'avérerait central ?). Si je décide au contraire de répondre à sa sollicitation, je sais d'emblée que ce sera au risque de me perdre...

En relisant des contributions parues dans Ethnologie française, je réalise que l'obstacle de la note y est d'autant plus fort que la revue est, depuis son origine en 1971, restée fidèle à la note de fin de texte. Avant les mises en forme automatiques des logiciels modernes, tout lecteur (disposant d'un minimum de sens typographique) pouvait comprendre cette relégation technique. Mais, depuis les années 2000, cet anachronisme,

faire une étude critique » car avec au total 87 notes cette contribution est en tête de l'échantillon que j'ai considéré. 
cette survivance, ne fait que rendre plus sensible l'errance qui menace toute personne consciencieuse.

Mettons en récit cette expérience ordinaire qui n'a, peut-être, jamais été contée : pour cela, imaginez que je suis bien installé à mon bureau et que je découvre un article de la dernière livraison d'Ethnologie française. Que j'avance avec entrain dans ma lecture en appréciant l'analyse subtile qui éclaire avec brio une situation sociale, un phénomène culturel. Et soudain, que je m'arrête. Ou plutôt, qu'un appel de note m'arrête. J'avais jusqu'alors négligé ces annexes que sont les notes, mais cette fois l'objet abordé me semble trop déterminant. Abandonnant le fil du texte principal, je feuillette donc rapidement les pages qui suivent, arrive à une nouvelle contribution, et de là, remonte le courant car je sais qu'avant la Zusammenfassung qui clôt l'article, avant l'abstract qui la précède, avant les références bibliographiques, se trouvent les Notes. J'y suis enfin. Je pourrais souffler, mais patatras, je trébuche : j'ai perdu en chemin le numéro de la note. C'est qu'au geste presque mécanique de tourner les feuilles doit succéder une mobilisation active de la mémoire. Or le temps écoulé frise les limites de ma mémoire immédiate. Je trébuche donc en piochant un mauvais numéro, voire en oubliant ce que je cherchais. Mon attention s'égare, vacille, et se rétablit in extremis : Ah oui, c'était la note 27 : la voilà ! Selon les cas, je jugerai si ce détour en valait la peine, mais il me faudra, de toutes façons, revenir sur mes pas, car jamais une lecture ne s'arrête dans l'impasse d'une note. Se déroule peut-être alors le moment le plus délicat de cette micro-aventure. À quelle page en étais-je ? Et sur cette page, où se cache le minuscule chiffre d'où je suis parti ? Si l'appel me disait où aller chercher, la note ne me renvoie plus nulle part ${ }^{3}$. Après avoir trébuché, je m'expose à tomber dans la dystopie d'un discours dont le fil serait rompu.

\footnotetext{
${ }^{3}$ Dans ses premières années, Ethnologie française regroupait dans ses « Notes » finales les commentaires et les éléments bibliographiques. Une référence appelée par exemple une première fois sous le renvoi « 9 » pouvait réapparaître plus loin dans le texte soit sous le même numéro de renvoi, soit avec un nouveau numéro (la note correspondant se contentait alors de rappeler le nom de l'auteur accompagné de «op. cit. », à charge pour le lecteur de retrouver la mention initiale...). Ce n'est, je crois, qu'à partir de 1990 que le départ fut fait entre les « notes » et les « références bibliographiques ».
} 
On ne formule habituellement pas cette expérience liminale de lecteur. Après tout, je n'avais qu'à être plus attentif, à être plus sélectif dans le choix des notes à consulter et tout cela ne m'a d'ailleurs pris que quelques secondes... Mais imaginez, dans la carrière d'un lecteur, le nombre d'heures que cela représente, et le poids des frustrations ainsi accumulées...

Pourtant, régulièrement, nous nous laissons attraper par ce nombre en exposant qui annonce une énigme. Vers quel horizon pointe-t-il ? Au fil du texte, on peut s'arrêter sur chaque note et, chaque fois, espérer une remarque profonde, un commentaire croustillant, une trouvaille bibliographique. On peut chiner à travers les notes comme d'autres courent les brocanteurs ou les bouquinistes. Exercice critique, la note est donc aussi une pratique ludique où le dévoilement de la petite intrigue qu'elle pose ne se réalise qu'en tournant les pages. Dans leur rôle de joueurs, certains lecteurs n'hésitent d'ailleurs pas à s'arranger avec la règle typographique. Nous le faisons tous, presque inconsciemment, en balayant du regard les notes infrapaginales des autres revues savantes. Dans les fins de texte d'Ethnologie française, la triche se fait plus raisonnée, plus stratégique : elle consiste, une fois arrivé à la fameuse note 27 , à vérifier si celles qui la précèdent ne présentaient pas quelque intérêt, et (pendant qu'on y est) à prendre un peu d'avance sur les suivantes.

Nul ne rougira cependant de ces grivèleries, car la note de fin de texte s'avère à l'expérience un adversaire redoutable. Immobile, elle guette les voyageurs qui passent à sa portée en affectant la plus petite forme qui soit, mais nombreux sont ceux qu'elle a ainsi égarés en chemin. Il faut pourtant, parfois, savoir répondre à son défi, la suivre jusqu'en son territoire, puis l'ayant maîtrisée, lui ayant subtilisé quelque trésor, retrouver le fil de sa lecture et poursuivre l'aventure. 